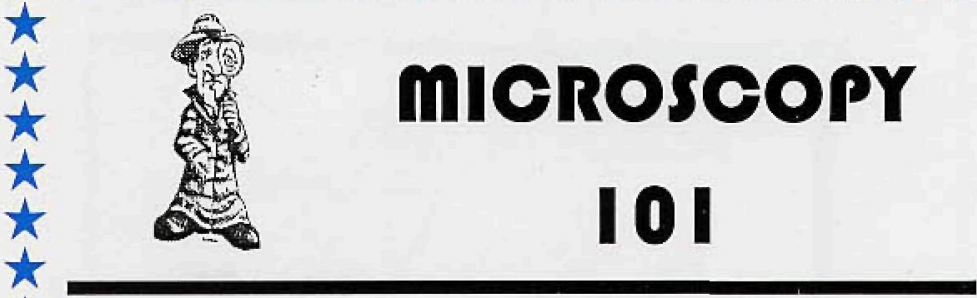

We appreciate the response to this publication feature - and welcome all contributions. Contributions may be sent to Phil Oshel, our Technical Editor at: Mr. Phil Oshel Tel.: (608)833-2885 PO Box $620068 \quad$ Fax: $(608) 836-1969$ Middleton WI 53562 eMail: peoshel@facstaff.wisc.edu

\section{Tips on Sectioning Polyethylene}

We have been sectioning polyethylene (PE) for over thitty years in our laboratory. Thin sectioning unpigmented polyethylene is not greatly different from sectioning biological samples.

There are several things that are going to be very important for this:

1) PE sectioning can not be done at room temperature, but only by cryosectioning.

2) $P E$ is very soft, and the key is to do the sectioning with minimum distortion. That means diamond knives, and the smaller the angle the better. We usually use a $35^{\circ} \mathrm{knife}$ angle.

t 3) PE is either injection molded (into parts) or melt extruded t (into film). The results obtained from sectioning along the injecA tion or extrusion direction might not necessarily be the same as when sectioning transverse to that direction. So give some thought as to which view would give the most useful information $\lambda$ considering the objectives of the study. Then it would be most timportant to always section all samples at the same angle relative $\uparrow$ to the machine direction. Otherwise, differences in section angle could be interpreted as differences between samples but such t would be false interpretations.

4) Pure unfilled polyethylenes are normally pretty unremarkt able by thin section TEM because there is nothing really to see or to give any contrast. Unless one is trying something exotic with the sections, such as negative staining of the lamellar or spherulitic structures, the main concern would be in the sectioning of the th inorganic additive particles which in general would be much harder than the polyethylene. The presence of these additives $\uparrow$ would impart small damage points on the knife edge, which would $\widehat{t}$ then show up as annoying striations in the final section (and image). Therefore, don't use good (and expensive) life science $\downarrow$ knives for such sectioning, but instead use a good materials scit ence knife (which can be obtained through SPI or the other firms t offering diamond knives.

5) We have found that when comparing microtomes from the two major ultramicrotome manufacturers, one can get comparathe results using either system. Just remember that for this kind t of work, a good cryosectioning system is needed.

ћ Charles A. Garber, Structure Probe, Inc.

\section{Cracking Hairs}

Recently, a request for a method to enable the cracking of human hair came up on the microscopy listserver. I have been involved with this type of problem many times, so set out below is a method which we have found works well both for light $\lambda$ microscopy and SEM. We have even used the technique $t$ to investigate failures in freezer bags.

1) Drill two or three holes through two SEM stubs placed face to face.

2) Pass the fibres through the holes.

3) Fix them in place with a water based carbon solu- th tion, making sure the fibre -- stub interface is well wetted.

4) When fully dry carefully place in liquid nitrogen.

5) When the bubbling stops lift out, again using care with the liquid nitrogen.

6) Place on an insulating surface and with a blade $t$ strike the interface between the two stubs. The system will th fracture.

7) When dried off, there are two sets of surfaces to look at using LM or SEM.

It works great on a number of differing media other $t$ than fibres., the only snag is that they should not be af- $t$ fected by the water base.

Steve Chapman, Pro-Train www.emcourses.com

\section{A Comment on Adhesive Tabs Cracking in Sputter Coaters}

We had noticed some time ago that our carbon adhesive tabs seemed to develop a series of fine cracks (looks like drying mud) after being sputter-coated. This was not a real problem, just a minor aesthetic annoyance when they $\lambda$ sometimes showed up in the background of a micrograph. However, when a client recently expressed concern about these cracks and wondered if sputtering was also damaging her samples, I did a rather unscientific series of tests to see what was going on.

If anyone is interested, we discovered that sputtering at $20 \mathrm{~mA}$ for 60 seconds invariably caused cracking of the tabs, but sputtering at $5 \mathrm{~mA}$ for 60 seconds did not. Sputtering for 20 seconds at $20 \mathrm{~mA}$ also avoided the cracks. To see if heating was the culprit, we put an uncoated tab on an SEM stub into a $60^{\circ} \mathrm{C}$ oven for an hour.

No cracks. We also ran one through the sputter coater cycle, but did not actually apply a coating, to see if the turbopump level vacuum alone would do it. No cracks.

So, if cracked tabs are keeping you awake at night, use a lower sputtering current or a shorter time.

Randy Tindall, University of Missouri TindallR@missouri.edu

\section{A Trick With Tripod Polishing}

A trick which I have found useful during tripod polishing is to use a $1 / 4$ " thick mirror instead of a clear glass on the polishing wheel. The mirror allows you to easily see if there are any contamination particles on the surface before placing the polishing disk on the glass. I got mine made at the local glass company for $\$ 6.50$. I specifically asked them to insure that there were not any scratches on the surface.

Kim W. Pierson, University of Wisconsin piersokw@uwec.edu 